\title{
Flora del bosque de encino (Quercus: Fagaceae) de dos barrancas de la ciudad de Puebla, México
}

\section{Oak forest flora (Quercus: Fagaceae) of two ravines of the Puebla city, Mexico}

\author{
Víctor Gutiérrez Pacheco ${ }^{*}$, Sonia Emilia Silva Gómezl y Laura Leonor Varela Olguín²
}

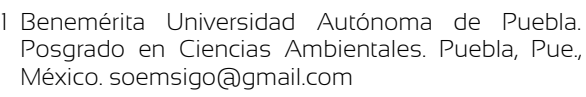

\author{
2 Benemérita Universidad Autónoma de Puebla \\ Preparatoria Enrique Cabrera Barroso (Urbana). \\ Puebla, Pue., México. Iaureh.gom(a)gmail.com
}

* Autor de correspondencia. v.guty@yahoo.com.mx

\section{RESUMEN}

Las barrancas de la ciudad de Puebla presentan un relicto de bosque de encino (Quercus: Fagaceae) que se encuentra sometido a fuertes presiones humanas. A pesar de la importancia que dicho relicto representa para una urbe carente de zonas arboladas, no existe información sobre su riqueza, diversidad y afinidad geográfica. De ahí los objetivos de este trabajo, inventariar los componentes de la flora vascular, determinar los patrones de distribución geográfica actual de las familias, géneros y especies, así como precisar la riqueza, diversidad y similitud florística de las barrancas El Conde y Malinalli. Se hicieron recorridos de campo y colecta de ejemplares botánicos, uso de los índices de Margalef, de Pielou y de Simpson para estimar los patrones de diversidad alfa y del índice de Similitud de Sorensen para determinar la diversidad beta. Se obtuvo una lista de 105 especies, agrupadas en 81 géneros y 40 familias, y ambas barrancas tienen una diversidad alfa y beta de mediana a alta. Por los servicios ecosistémicos que los espacios arbolados proporcionan a las zonas urbanas, se deberá ahondar en el conocimiento del bosque y ampliar el estudio hacia otras barrancas como un paso hacia su recuperación, remediación, manejo y conservación.

PALABRAS CLAVE: biodiversidad, ecosistema, especies nativas, índice, inventario, servicios ecosistémicos.

\section{ABSTRACT}

The Puebla city ravines have a relic of oak forest (Quercus: Fagaceae) that is subject to strong human pressures. Despite the importance that this relict represents for a city lacking in wooded areas, there is no information on its richness, diversity, and geographical affinity. Hence the objective of this work, to carry out an inventory of the vascular flora components, determining the current geographic distribution patterns of the families, genera, and species, and specify the richness, diversity, and floristic similarity of El Conde and Malinalli ravines. This was done through field trips and botanical specimen collection, using the Margalef, Pielou and Simpson indices to estimate alpha diversity patterns and the Sorensen Similarity Index to determine beta diversity. A list of 105 species was obtained, grouped into 81 genera and 40 families, and both ravines have a medium to high alpha and beta diversity. Because to the ecosystem services that wooded areas provide to urban areas, knowledge of the forest should be deepened, and the study extended to other ravines as a step towards recovery, remediation, management, and conservation.

KEYWORDS: biodiversity, ecosystem, native species, index, inventory, ecosystem services. 


\section{INTRODUCCIÓN}

Los bosques de encino (Quercus) son comunidades vegetales, que se distribuyen ampliamente en áreas de clima templado y semihúmedo de México, encontrándose principalmente entre $1200 \mathrm{~m}$ y $2800 \mathrm{~m}$ s.n.m. (Rzedowski, 1988). Se reconocen dos centros de diversidad de encinos en el mundo, uno en Asia y otro en América (Nixon, 2009), siendo este último el más grande y situado en México (Nixon, 1993; Valencia, 2010). Los encinares son muy comunes en el Eje Volcánico Transversal (EVT), aunque muy impactados por la actividad humana ya que ocupaban regiones de clima atractivo para la ocupación humana y áreas favorables para la agricultura (Rzedowski, 1988).

De acuerdo al Inventario Estatal Forestal y de Suelos, la zona donde se extiende la ciudad de Puebla se presenta como asentamiento humano sin un tipo de ecosistema característico y las áreas aledañas que la circundan se consideran no forestales, con excepción del área hacia el este de la ciudad, que aparece como de tipo de vegetación de coníferas y latifoliadas, con presencia de bosque de encino restringido a barrancas, laderas y lomeríos en varios estados de sucesión que indican los severos procesos de degradación que han sufrido (Secretaría de Medio Ambiente y Recursos Naturales [Semarnat] y Comisión Nacional Forestal [Conafor], (2013).

Sin embargo, dentro de la mancha urbana propiamente, no se reconoce ya la presencia de bosque de encino y solo se aceptan como zonas arboladas y áreas verdes a aquellos parques, jardines y zonas de vegetación irreductible que son espacios con vegetación inducida sobre todo de especies exóticas (Ayuntamiento de la ciudad de Puebla, 2016).

Una característica de la ciudad de Puebla es la presencia de un sistema de barrancas que la entrecruza; las cuales son formaciones geológicas que tienen su origen en el vulcanismo y en la erosión hídrica de escurrimientos pluviales provenientes de la montaña Malinche (Instituto de Geografía-UNAM, 1989). En muchos sitios, a lo largo de las mismas, se aprecia relicto de bosque de encino. Entonces, este es un bosque urbano por encontrarse dentro de los límites de la ciudad, aunque no está reconocido como tal pues no forma parte del inventario de las áreas verdes de la ciudad (Ayuntamiento de la ciudad de Puebla, 2016), y es un bosque natural conformado por árboles, arbustos y herbáceas asociadas.

A pesar de que las barrancas están siendo fuertemente impactadas por la actividad humana en acciones como asentamiento humanos irregulares, disposición de aguas residuales sin tratamiento y vertido de residuos sólidos entre otras, desde un punto de vista fisionómico, aún se puede observar la presencia de su componente forestal que contrasta fuertemente con el entorno construido en una ciudad carente de vegetación y deficiente en el equipamiento de áreas verdes (International City/County Management Association [ICMA], 2017).

La condición de espacios arbolados permite a las barrancas proveer múltiples beneficios al contexto citadino, toda vez que mejoran la calidad del ambiente y la salud humana, como el abatimiento de la contaminación, la recarga de acuíferos, la regulación de inundaciones, la regulación del clima, la protección a otros recursos, el refugio a la fauna silvestre, la recreación y la salud mental y la arquitectura del paisaje (Nowak, Crane y Stevens, 2006).

Por los servicios enunciados y bajo el principio de que la descripción cualitativa es un primer paso en la evaluación de una comunidad arbórea (Spurr, 1964), se considera pertinente conocer la composición del bosque de las barrancas dada la escasa o nula información disponible. Todo en aras de acceder a su revaloración, recuperación, manejo y conservación que posibiliten el que continúen proporcionando sus servicios a la urbe, manteniendo y mejorando el ambiente y la salud humana.

\section{OBJETIVOS}

Realizar un listado e inventario florístico del bosque de las barrancas El Conde y Malinalli. Así mismo, determinar los patrones de diversidad alfa de las comunidades de ambas barrancas a través de los índices de Margalef, de Pielou y de Simpson y de diversidad beta a través del índice de Similitud de Sorensen. 


\section{MATERIALES Y MÉTODOS}

\section{Área de estudio}

El trabajo se desarrolló en dos barrancas de la ciudad de Puebla: El Conde y Malinalli. El Conde se ubica en el norte de la urbe (Fig. 1), a un costado de la vía corta a Santa Ana Chiautempan, Tlaxcala, en las coordenadas $19^{\circ} 09^{\prime} 80^{\prime}$ " de latitud norte y el meridiano $98^{\circ} 18^{\prime} 74$ " de longitud oeste, a $2680 \mathrm{~m}$ s.n.m. El suelo es de tipo Luvisol y el clima es templado subhúmedo con lluvias en verano, de mayor humedad. Los valores promedio anuales de temperatura y precipitación son $16{ }^{\circ} \mathrm{C}$ y 800 $\mathrm{mm}$, respectivamente (Instituto Nacional de Estadística y Geografía [Inegi], 2010).
La barranca $\mathrm{El}$ Conde discurre en una zona entre áreas habitacionales hacia el Norte y el parque industrial 5 de Mayo hacia el sur. Tiene una longitud de $1700 \mathrm{~m}$ con un ancho que va desde los $30 \mathrm{~m}$ en algunos sitios a los $100 \mathrm{~m}$ en otros. En algunos lugares no se puede precisar la amplitud pues los referentes se han perdido por la presencia de casas-habitación. En cuanto a su profundidad, varía en los diferentes sitios por donde se prolonga, desde $60 \mathrm{~m}$ en las zonas más profundas hasta $0 \mathrm{~m}$ en donde por obras de relleno, presencia de casas en sus orillas y pavimentación de una calle que se habilitó en la misma, quedó a ras de suelo. Presenta asentamientos humanos irregulares en las zonas más accesibles, descargas de aguas residuales domésticas de las colonias circundantes y las provenientes del parque industrial 5 de Mayo con el que colinda.
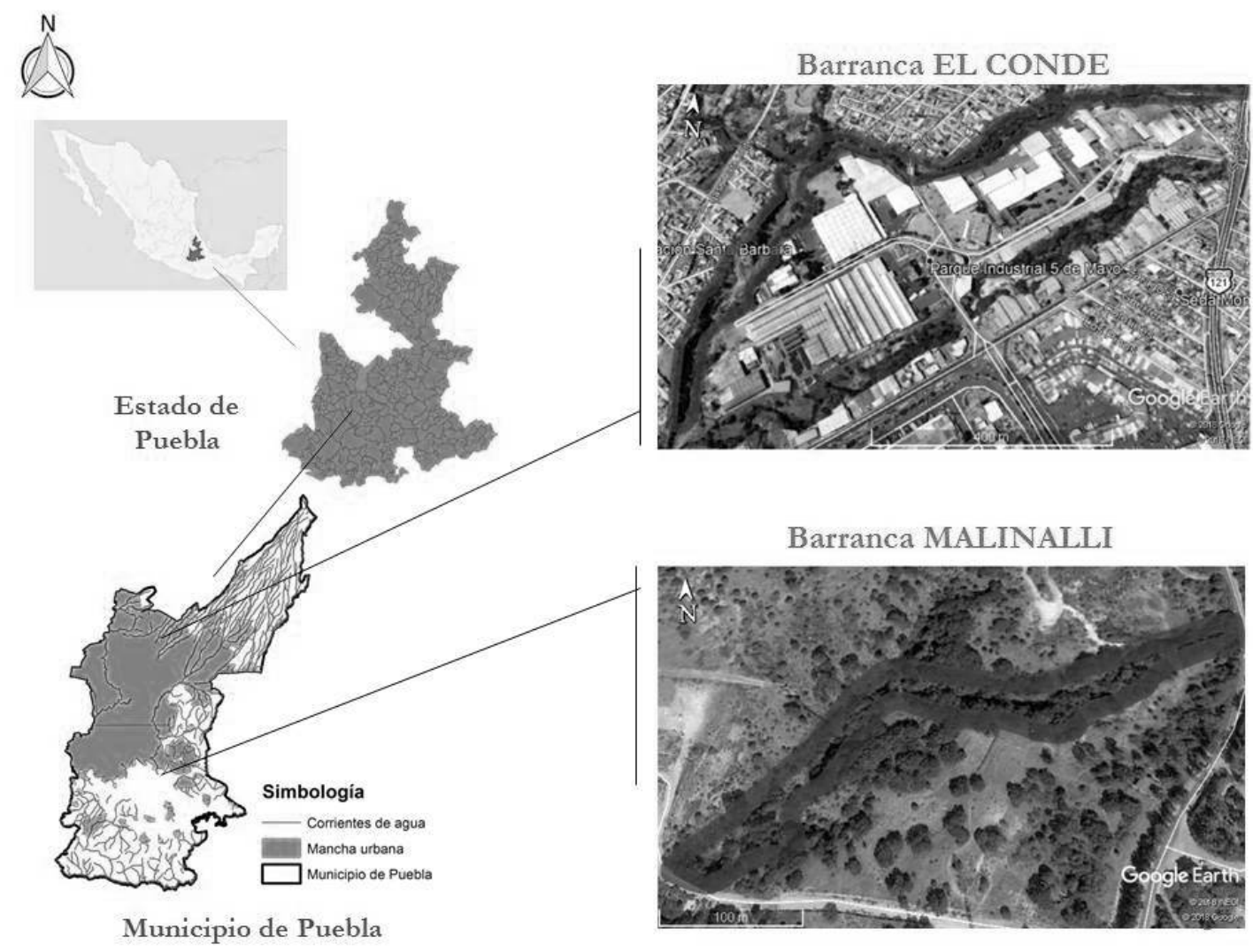

FIgura 1. Ubicación de las barrancas en estudio en la ciudad de Puebla, México. 
La barranca Malinalli se sitúa al sur de la ciudad (Fig. 1), en las coordenadas $18^{\circ} 93^{\prime} 93^{\prime \prime}$ de latitud norte y el meridiano $98^{\circ} 13^{\prime} 68^{\prime \prime}$ de longitud oeste y a una altura de $2060 \mathrm{~m}$ s.n.m. El suelo es de tipo Durisol y el clima es templado subhúmedo con lluvias en verano, de humedad media. La temperatura y precipitación promedio anuales son $18{ }^{\circ} \mathrm{C}$ y $500 \mathrm{~mm}$, respectivamente (Inegi, 2010). Se ubica al Este del zoológico Africam Safari en los límites de la mancha urbana y no presenta asentamientos humanos e impactos antrópicos evidentes. Tiene una longitud de $750 \mathrm{~m}$ aproximadamente y su profundidad varía a lo largo de su recorrido, desde $1.3 \mathrm{~m}$ hasta $8 \mathrm{~m}, 10 \mathrm{~m}$ o $12 \mathrm{~m}$ en otros sitios. En cuanto a la amplitud también hay variaciones, desde $8 \mathrm{~m}$ en los sitios más estrechos hasta $50 \mathrm{~m}$ en los puntos más amplios.

\section{Trabajo de campo y determinación de especies}

En los periodos de secas y de lluvias de los años 2017 y 2018 se realizaron salidas de campo para recolectar material de herbario, el cual se procesó mediante las técnicas de recolección y preparación de ejemplares botánicos descritas por Lot y Chiang (1986), y se determinó la abundancia de las especies de diagnóstico a través del conteo de especímenes. Por lo pequeño de las áreas de las barrancas, no fueron necesarias técnicas de muestreo, pudiéndose inventariar en su totalidad.

De los ejemplares recolectados, se obtuvieron los datos del tipo de vegetación donde se desarrollan: bosque de Quercus y vegetación secundaria producto de la remoción y posterior regeneración de este bosque. La forma biológica o hábito de crecimiento se determinó de acuerdo con los criterios de Font (2000). La clasificación considera categorías distribuidas en tres atributos distintos: hábito (árbol, arbusto y hierba); hábitat (epífito y terrestre) y tipo de nutrición (autótrofa, hemiparásita y holoparásita).

La identificación taxonómica se realizó con el uso de claves taxonómicas, el apoyo de especialistas y bibliografía especializada (floras y monografías): Flora fanerogámica del Valle de México (Calderón de Rzedowski y Rzedowski, 2001), Lista florística comentada de plantas vasculares silvestres en San Juan Quetzalcoapan, Tlaxcala, México (Vibrans, 1997) y Plantas Silvestres de Puebla (Rodríguez, Coombes y Jiménez, 2009). Los ejemplares determinados se cotejaron con los de la colección del HUAP (Herbario de la Benemérita Universidad Autónoma de Puebla), donde se depositó un duplicado del material de herbario; otro duplicado se depositó en el Departamento Universitario para el Desarrollo Sustentable de la misma institución.

La composición florística se describió mediante una lista ordenada por grupos taxonómicos y de manera alfabética. Las abreviaturas de los autores se citaron conforme a Villaseñor, Ortiz y Redonda-Martínez (2008). Para la nomenclatura de los géneros y las especies se hizo uso de la base de datos The Plant List (2013) y cuando el nombre binario se indicó como no resuelto se usó el nombre indicado en el sitio web Trópicos del Missouri Botanical Garden (2014). Las Angiospermas (Magnoliophyta) se agruparon en las clases taxonómicas Liliopsida (monocotiledoneas) y Magnoliopsida (Dicotiledoneas) de acuerdo con la propuesta de Cronquist (1981). Las familias se ordenaron según los sistemas de clasificación propuestos por Mickel y Smith (2004) para Pteridophyta, McVaugh (1992) para Gimnospermophyta y del Angiosperm Phylogeny Group [APG IV] (2016) para Magnoliophyta. El origen y la distribución geográfica de las especies (nativa o introducida) se determinaron a través de la consulta del sitio web Trópicos del Missouri Botanical Garden (2014), Malezas de México (Vibrans, 2006) y la Encyclopedia of Life (EOL) (National Museum of Natural History $[\mathrm{NMNH}], 2019)$. Se usaron las categorías fitogeográficas: endémica de México $(\mathrm{Mx})$, México a Centroamérica (Mx-cAm), México a Norteamérica (Mx$\mathrm{nAm})$, Norteamérica a Centroamérica (nAm-cAm), México a Sudamérica (Mx-sAm) y Norteamérica a Sudamérica (Am) (Zepeda, Burrola, White y Rodríguez, 2017), así como las categorías Megaméxico 1, 2 y 3 (Rzedowski, 1991a).

\section{Análisis de la información}

La diversidad alfa se estimó mediante la riqueza específica y la estructura de la comunidad. Para la primera se hizo uso 
del índice de Margalef $\left(\mathrm{D}_{\mathrm{mg}}\right)$ y para la segunda de los índices de Equidad de Pielou $\left(J^{\prime}\right)$ y de Simpson ( $\lambda$ ) (índice de dominancia), mediante las ecuaciones $1,2 \quad$ y 3 respectivamente (Moreno, 2001). Conforme a los supuestos del método fitosociológico y a la escuela norteamericana de aproximación a la vegetación, se consideraron como especies de diagnóstico a las especies leñosas (Alcaraz, 1999). De las especies de diagnóstico, se consideraron los individuos enraizados dentro de la zona de estudio, con un DAP $\geq 5 \mathrm{~cm}$ (Sánchez-Rodríguez, López-Mata, GarcíaMoya y Cuevas-Guzmán, 2003) y una altura mayor a $2.5 \mathrm{~m}$.

$$
\begin{gathered}
D_{m g}=(S-1) / \ln (\mathrm{N}) \\
J^{\prime}=H^{\prime} / H_{\max } \\
\lambda=\sum_{i=1}^{S} P i^{2} \\
H_{\text {max }}^{\prime}=\ln S \\
H^{\prime}=-\sum_{i=1}^{S} P i(\ln P i) \\
P i=n i / N
\end{gathered}
$$

Donde:

$S=$ número de especies

$N=$ número de todos los individuos de todas las especies

$\mathrm{Pi}=$ proporción de individuos de la especie $i$ respecto al total de individuos $N$ (es decir la abundancia relativa de la especie i)

$n i=$ número de individuos de la especie $i$ (Moreno, 2001)

El índice de Margalef se basa en determinar la biodiversidad a través del número de especies presentes en el sitio, mientras que los índices de equidad de Pielou y de Simpson se basan en la abundancia relativa de los individuos, que determina la importancia de cada especie.

La diversidad beta se determinó de acuerdo con el índice de Similitud de Sorensen $\left(I_{s}\right)$ mediante la ecuación (7), entendiéndose que mientras menos especies compartan mayor será su beta diversidad. (Moreno, 2001).

$$
I_{s}=2 c / a+b
$$

Donde:

$\mathrm{a}=$ número de especies presentes en el sitio $\mathrm{A}$

$\mathrm{b}=$ número de especies en el sitio $\mathrm{B}$

$\mathrm{c}=$ número de especies presentes en ambos sitios $\mathrm{A}$ y $\mathrm{B}$

\section{Resultados}

\section{Riqueza y composición florística}

La flora registrada para la zona de estudio se compone de 105 especies, 81 géneros y 40 familias de plantas vasculares (Tabla 1). De estas especies, 64 se hallaron en la barranca Malinalli y 71 en la barranca El Conde. Las dicotiledóneas (Magnoliópsida) representaron más de $80 \%$ de las familias, géneros y especies (Tabla 2). Siete familias concentraron $47 \%$ de la flora, destacándose las familias Asteraceae, Fabaceae y Fagaceae por presentar el mayor número de especies y Asteraceae y Fabaceae el mayor número de géneros (Fig. 2). La familia Asteraceae sobresale ya que incluye 15\% de los géneros y $13 \%$ de las especies de la flora analizada. Los géneros con el mayor número de especies fueron Agave (3 especies), Commelina (3), Ipomoea (5) y Quercus (8) (Tabla 1).

\section{Hábito, hábitat y tipo de nutrición}

Respecto al hábito de crecimiento, las hierbas representaron un total de 58 especies (55\%), los árboles con 21 especies y los arbustos con 26 especies, representaron $20 \%$ y $25 \%$, respectivamente. En cuanto al hábitat, $97.1 \%$ de las especies fueron terrestres y tres $(2.9 \%)$ epífítas. Conforme al tipo de nutrición, $95.2 \%$ de las especies fueron autótrofas, mientras que 4.8\% fueron parásitas. De estas últimas, se registraron cuatro hemiparásitas $(3.8 \%)$ y una holoparásita (1\%) (Tabla 1).

\section{Tipos de vegetación}

Del total de especímenes recolectados para toda la zona de estudio, 64 especies (61\%) fueron propias del bosque de Quercus, mientras que $32(30.5 \%)$ se encontraron en vegetación secundaria y nueve $(8.5 \%)$ tanto en bosque de Quercus como en vegetación secundaria. 
TABLA 1. Lista de especies del bosque de Quercus de las barrancas urbanas El Conde y Malinalli de la ciudad de Puebla, México.

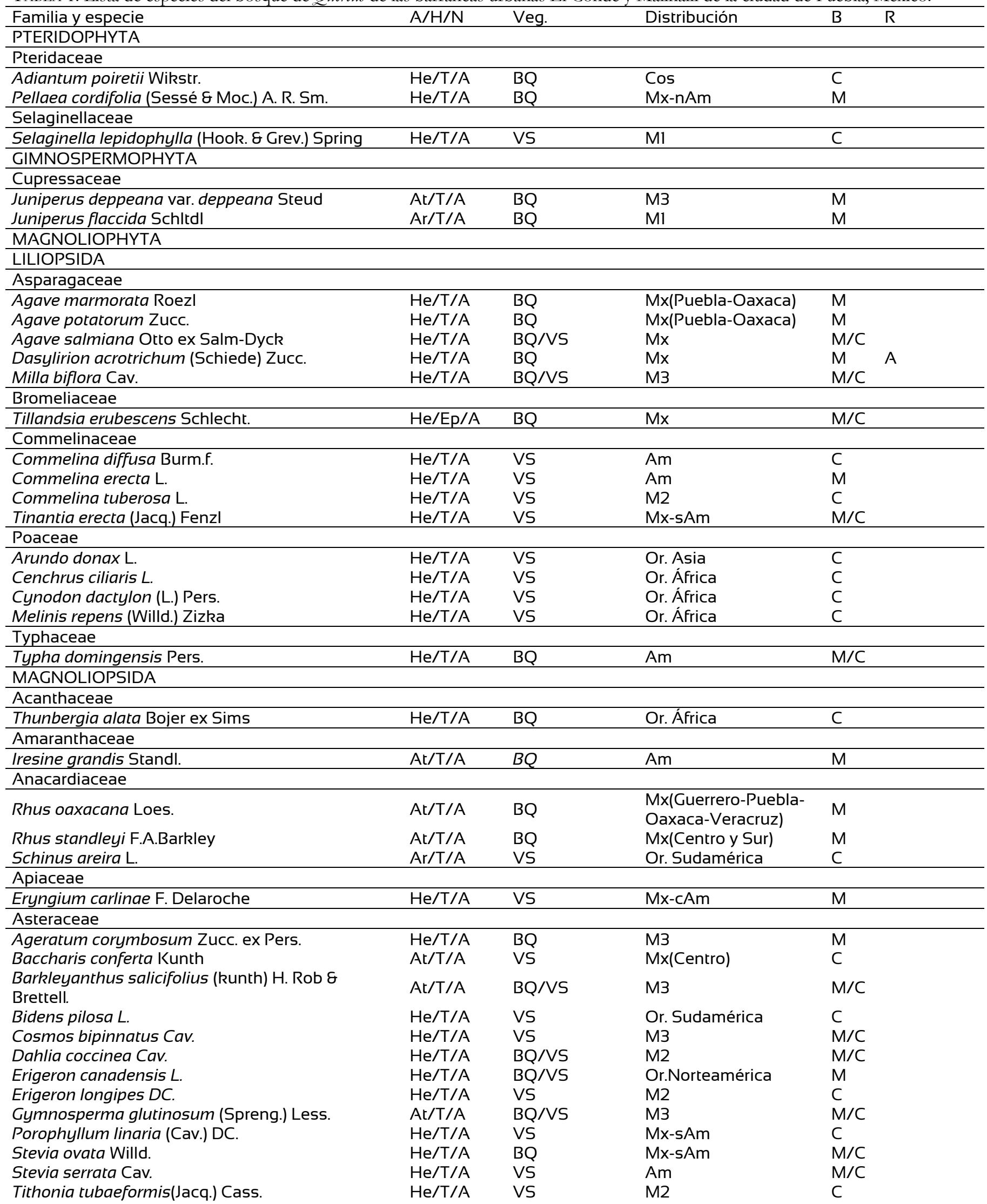


TABLA 1. Lista de especies del bosque de Quercus de las barrancas urbanas El Conde y Malinalli de la ciudad de Puebla, México.

\begin{tabular}{|c|c|c|c|c|c|}
\hline Familia y especie & $\mathrm{A} / \mathrm{H} / \mathrm{N}$ & Veg. & Distribución & B & R \\
\hline Zinnia peruviana (L.) L. & $\mathrm{He} / \mathrm{T} / \mathrm{A}$ & BQ/VS & Am & $\mathrm{M} / \mathrm{C}$ & \\
\hline Begonia gracilis Kunth & $\mathrm{He} / \mathrm{T} / \mathrm{A}$ & $\mathrm{BQ}$ & $\mathrm{Mx}$ & C & \\
\hline Cordia curassavica (Jacq.) Roem. \& Schult. & $\mathrm{At} / \mathrm{T} / \mathrm{A}$ & BQ & Mx-sAm & M & \\
\hline Wigandia urens (Ruiz \& Pav.) Kunth & $\mathrm{At} / \mathrm{T} / \mathrm{A}$ & VS & Mx-sAm & $\mathrm{C}$ & \\
\hline \multicolumn{6}{|l|}{ Cactaceae } \\
\hline Opuntia streptacantha Lem. & $\mathrm{At} / \mathrm{T} / \mathrm{A}$ & $\mathrm{BQ}$ & Mx(Centro) & $\mathrm{M}$ & \\
\hline \multicolumn{6}{|l|}{ Convolvulaceae } \\
\hline Ipomoea batatoides Choisy & $\mathrm{He} / \mathrm{T} / \mathrm{A}$ & BQ & Mx-sAm & $\mathrm{M} / \mathrm{C}$ & \\
\hline Ipomoea orizabensis (Pelletan) Ledeb. Ex Steud. & $\mathrm{He} / \mathrm{T} / \mathrm{A}$ & BQ & M2 & $\mathrm{M} / \mathrm{C}$ & \\
\hline Ipomoea purga (Wender.) Hayne & $\mathrm{He} / \mathrm{T} / \mathrm{A}$ & BQ & $\mathrm{M} 2$ & $\mathrm{M} / \mathrm{C}$ & \\
\hline Ipomoea purpurea (L.) Roth & $\mathrm{He} / \mathrm{T} / \mathrm{A}$ & BQ & Mx-sAm & $M / C$ & \\
\hline \multicolumn{6}{|l|}{ Ericaceae } \\
\hline \multirow{3}{*}{$\begin{array}{l}\text { Arbutus xalapensis Kunth } \\
\text { Arctostaphylos pungens Kunth } \\
\text { Comarostaphylis polifolia (Kunth.) Zucc. ex } \\
\text { Klotzsch }\end{array}$} & $\mathrm{Ar} / \mathrm{T} / \mathrm{A}$ & BQ & M3 & M & \\
\hline & $\mathrm{At} / \mathrm{T} / \mathrm{A}$ & BQ & M1 & M & \\
\hline & $\mathrm{At} / \mathrm{T} / \mathrm{A}$ & $\mathrm{BQ}$ & Mx(Centro y Sur) & M & \\
\hline \multicolumn{6}{|l|}{ Euphorbiaceae } \\
\hline Acalypha phleoides Cav. & $\mathrm{He} / \mathrm{T} / \mathrm{A}$ & $\mathrm{BQ}$ & M3 & M & \\
\hline Ricinus communis L. & $\mathrm{At} / \mathrm{T} / \mathrm{A}$ & VS & Or. África & $\mathrm{C}$ & \\
\hline \multicolumn{6}{|l|}{ Fabaceae } \\
\hline Acacia angustissima (Mill.) Kuntze & $\mathrm{At} / \mathrm{T} / \mathrm{A}$ & BQ & nAm-cAM & C & \\
\hline Acacia schaffneri (S. Watson) F. J. Herm & $\mathrm{At} / \mathrm{T} / \mathrm{A}$ & BQ & M1 & M & \\
\hline Quercus acutifolia Née. & $\mathrm{Ar} / \mathrm{T} / \mathrm{A}$ & BQ & M2 & $M / C$ & \\
\hline Quercus castanea Née & $\mathrm{Ar} / \mathrm{T} / \mathrm{A}$ & BQ & $\mathrm{M} 2$ & C & \\
\hline Quercus crassipes Bonpl. & $\mathrm{Ar} / \mathrm{T} / \mathrm{A}$ & BQ & $M x$ & $\mathrm{M} / \mathrm{C}$ & \\
\hline Quercus deserticola Trel & $\mathrm{Ar} / \mathrm{T} / \mathrm{A}$ & BQ & Mx(Centro y Sur) & C & \\
\hline Quercus glaucoides M. Martens \& Galeotti & $\mathrm{Ar} / \mathrm{T} / \mathrm{A}$ & BQ & Mx(Centro y Sur) & $\mathrm{M} / \mathrm{C}$ & \\
\hline Quercus laurina Bonpl. & Ar/T/A & BQ & $\mathrm{M} 2$ & $M / C$ & \\
\hline Quercus mexicana Bonpl. & $\mathrm{Ar} / \mathrm{T} / \mathrm{A}$ & BQ & Mx & $M / C$ & \\
\hline $\begin{array}{l}\text { Quercus rugosa Née } \\
\text { Geraniaceae }\end{array}$ & $\mathrm{Ar} / \mathrm{T} / \mathrm{A}$ & BQ & M3 & $M / C$ & \\
\hline Geranium seemannii Peyr. & $\mathrm{He} / \mathrm{T} / \mathrm{A}$ & BQ & M2 & C & \\
\hline \multicolumn{6}{|l|}{ Lamiaceae } \\
\hline $\begin{array}{l}\text { Clinopodium macrostemum (Moc. \& Sessé ex } \\
\text { Benth.) Kuntze }\end{array}$ & $\mathrm{He} / \mathrm{T} / \mathrm{A}$ & BQ & Mx(Centro y Sur) & M & \\
\hline Leonotis nepetifolia (L.) R. Br & $\mathrm{He} / \mathrm{T} / \mathrm{A}$ & VS & Or. África & C & \\
\hline \multicolumn{6}{|l|}{ Loranthaceae } \\
\hline Psittacanthus calyculatus (DC,) G. Don & $\mathrm{He} / \mathrm{Ep} / \mathrm{He}$ & $\mathrm{BQ}$ & Mx-sAm & $\mathrm{M} / \mathrm{C}$ & \\
\hline \multicolumn{6}{|l|}{ Myrtaceae } \\
\hline Eucalyptus camaldulensis Dehnh. & $\mathrm{Ar} / \mathrm{T} / \mathrm{A}$ & VS & C (Or. Australia) & $\mathrm{C}$ & \\
\hline Eucalyptus globulus Labill. & $\mathrm{Ar} / \mathrm{T} / \mathrm{A}$ & $\mathrm{BQ}$ & C (Or. Australia) & M & \\
\hline \multicolumn{6}{|l|}{ Nyctaginaceae } \\
\hline Mirabilis jalapa $\mathrm{L}$. & $\mathrm{He} / \mathrm{T} / \mathrm{A}$ & VS & Mx-sAm & $\mathrm{C}$ & \\
\hline
\end{tabular}


TABLA 1. Lista de especies del bosque de Quercus de las barrancas urbanas El Conde y Malinalli de la ciudad de Puebla, México.

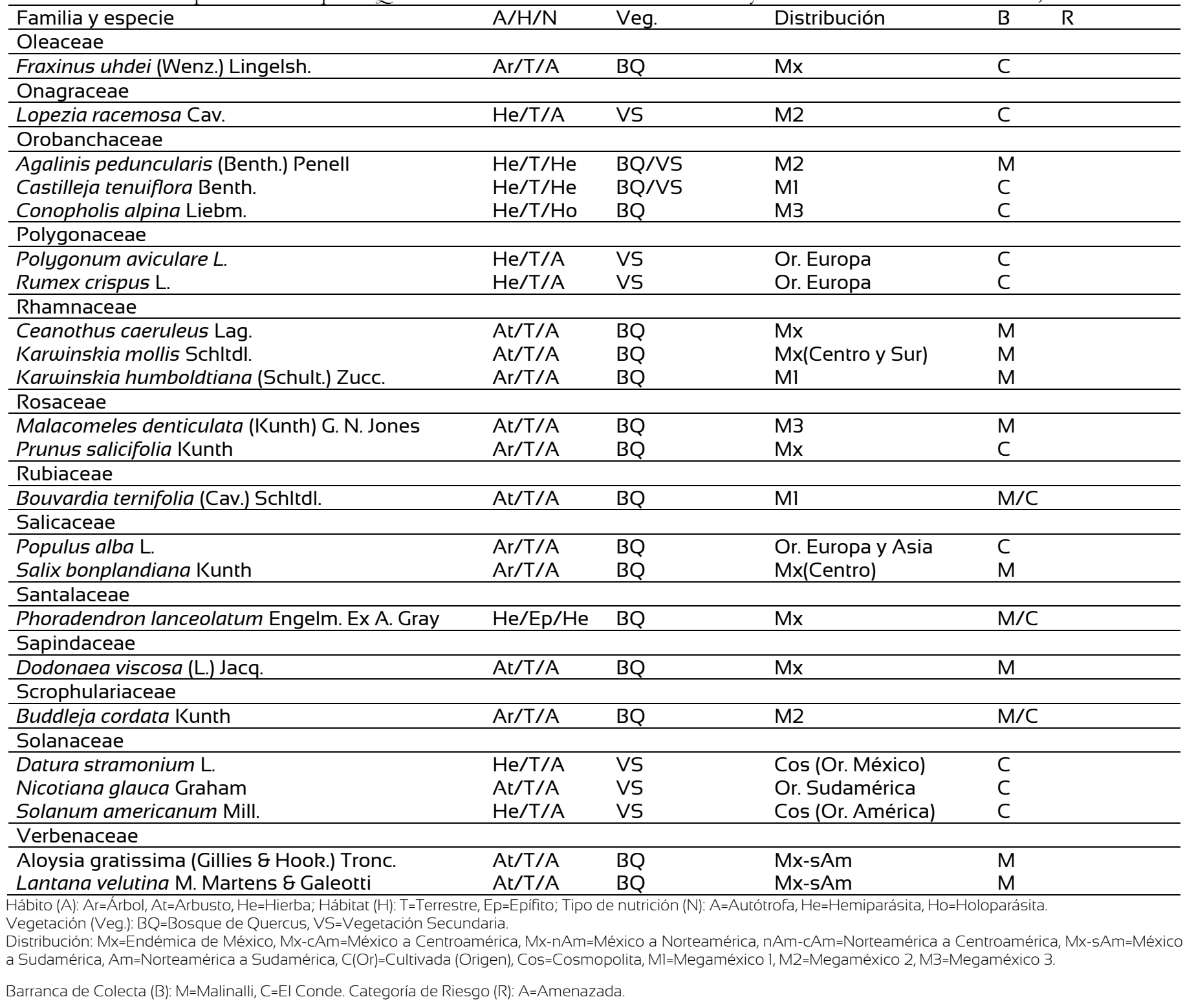

TABLA 2. Riqueza de plantas de las barrancas urbanas El Conde y Malinalli.

\begin{tabular}{llll}
\hline & Familias & Géneros & Especies \\
\hline Pteridofitas & $2(5 \%)$ & $3(3.7 \%)$ & $3(2.9 \%)$ \\
Gimnospermophyta & $1(2.5 \%)$ & $1(1.2 \%)$ & $2(1.9 \%)$ \\
Monocotiledoneas & $5(12.5 \%)$ & $11(13.4 \%)$ & $15(14.3 \%)$ \\
Dicotiledoneas & $32(80 \%)$ & $67(81.7 \%)$ & $85(80.9 \%)$ \\
Total & 40 & 82 & 105 \\
\hline
\end{tabular}




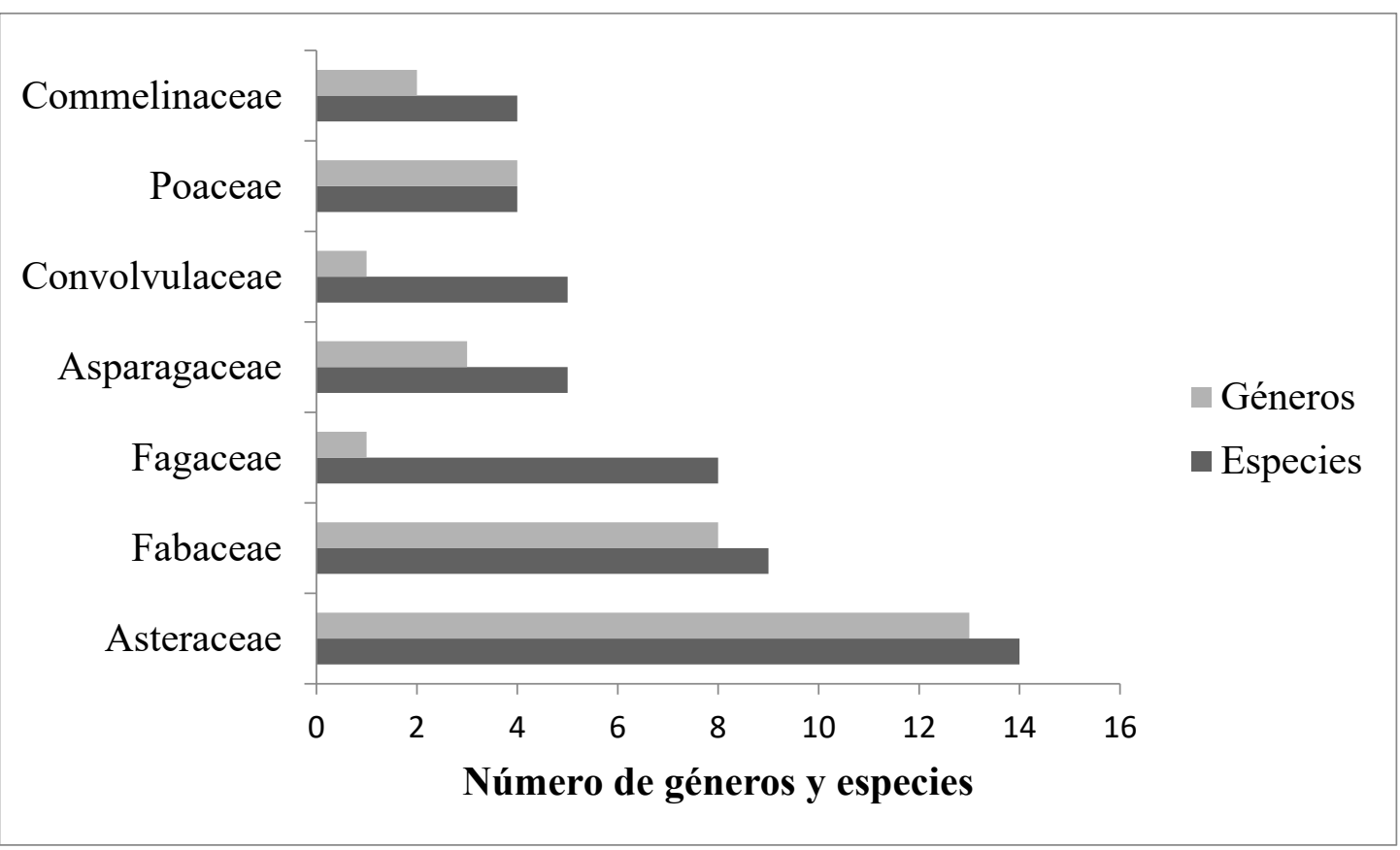

FIGURA 2. Número de especies y géneros de las familias más representativas del bosque de Quercus de las barrancas urbanas El Conde y Malinalli.

La barranca El Conde presentó el mayor número de especies de Quercus (8), en la barranca Malinalli se presentaron claros dentro del bosque los cuales están siendo habitados por especies vegetales propias de matorral xerófilo, como individuos de los géneros Opuntia, Mammilaria, Acacia y Agave.

\section{Afinidades geográficas y origen}

De las especies registradas en las dos barrancas, $85 \%$ son nativas, $59 \%$ se distribuye dentro de los límites biológicos y políticos de México (Megaméxico 3 según Rzedowski, 1991a), destacando las endémicas a los límites políticos de México (28\%). De la flora que se restringe a los límites políticos de México, 12 especies (11.4\%) son endémicas a las regiones centro y sur del país. Una especie es endémica, limitándose a los estados de Guerrero, Oaxaca, Puebla y Veracruz (Tabla 1); y dos son endémicas con una distribución restringida a los estados de Oaxaca y Puebla. Cuarenta y una de cada cien especies son de amplia distribución rebasando los límites políticos y biológicos de México (Megaméxico 3 según Rzedowski, 1991a); de estas, la mayor parte se comparten con Sudamérica $(12.3 \%)$ y con toda América (7.6\%) (Tabla 3).

En relación con la flora introducida, 14 especies (15\%) se encontraron en la barranca El Conde, que es la que tiene el mayor impacto humano, mientras que en la barranca Malinalli se registraron dos especies.

\section{Especies que ameritan atención especial}

Se encontraron dos especies (Dasylirion acrotrichum (Schiede) Zucc. y Erythrina coralloides DC.) enlistadas en la NOM-059SEMARNAT-2010 en la categoría de amenazadas (Secretaría de Medio Ambiente y Recursos Naturales [Semarnat], 2010). Se deben considerar también las tres especies endémicas locales ya mencionadas anteriormente.

\section{Diversidad}

La diversidad alfa de especies de acuerdo con los índices de Margalef, Pielou y Simpson fueron para la barranca Malinalli: 4.98, 0.85 y 0.07 , respectivamente, y para la barranca El Conde: 3.97, 0.94 y 0.15, respectivamente (Tabla 4). La mayor riqueza y diversidad de especies se 
encontró en la barranca Malinalli de acuerdo con los índices de Margalef y de Simpson, mientras que de acuerdo con el índice de Pielou, la barranca El Conde es la más diversa. La diversidad beta se determinó a través del índice de Sorensen, Treinta especies son comunes a ambas barrancas y de acuerdo con este índice, la similitud entre ambas barrancas es de 44\% (Tabla 4).

\section{Discusıón}

\section{Composición florística}

Las barrancas de la ciudad de Puebla no forman parte del polígono del Parque Nacional Malinche, aunque sí están inscritas en su zona de influencia, particularmente el área donde se encuentra la barranca El Conde, descrita como de bosque de Quercus (López-Domínguez y Acosta, 2005).

Existen diversos trabajos que describen la vegetación del Parque Nacional Malinche a partir del de Sosa (1927), quien investigó sobre la composición de los bosques de dicho parque. Sin embargo, no hay registros de trabajos para los relictos de bosque de la zona donde se asienta la ciudad de Puebla, por lo que este trabajo constituye un primer esfuerzo por acercarse al conocimiento de la flora existente en las barrancas urbanas y obtener un listado florístico de las mismas.

El listado da cuenta de una composición florística en la cual $56 \%$ de los géneros y $61 \%$ de las especies de la flora se concentran en 12 de las 40 familias registradas. Familias como Asparagaceae, Asteraceae, Fabaceae, Poaceae y Solanaceae agrupan un tercio de la flora de la zona de estudio; en concordancia, estas familias están entre las 15 más ricas y frecuentes de México (Villaseñor, 2003). Asteraceae presentó el mayor número de géneros y especies, lo que concuerda con su prevalencia en el país, donde es la familia de angiospermas más numerosa en cuanto a géneros y especies (Villaseñor, 2004, Villaseñor y Ortiz, 2014), también concuerda con el hecho de que la Faja Volcánica Transmexicana (FVTM), que es donde se ubica la zona de estudio, es centro de diversidad y endemismo de Asteraceae (Villaseñor y Ortiz, 2007).

A nivel de género, el mayor número de especies se presentó en Quercus (ocho especies, siete endémicas), Ipomoea (5 / 2), Commelina (3 / 1) y Agave (3 / 3). El predominio de especies de los géneros Quercus e Ipomoea tiene sentido en la medida en que: a) son de los diez géneros más diversos del país (Villaseñor, 2004), b) México es el principal centro mundial de diversificación y endemismos de encinos (Nixon, 1993; Valencia, 2004) y c) Quercus es cuantitativamente importante en la FVTM (Miranda, 1947). En cuanto a los valores por tipo de hábito: $63 \%$ de los géneros incluyen herbáceas, $55 \%$ arbustos y $25 \%$ árboles; en general hay una tendencia hacia el patrón señalado por Villaseñor (2004), para géneros de plantas vasculares de México.

TABLA 3. Síntesis de la distribución de las especies presentes en las barrancas El Conde y Malinalli.

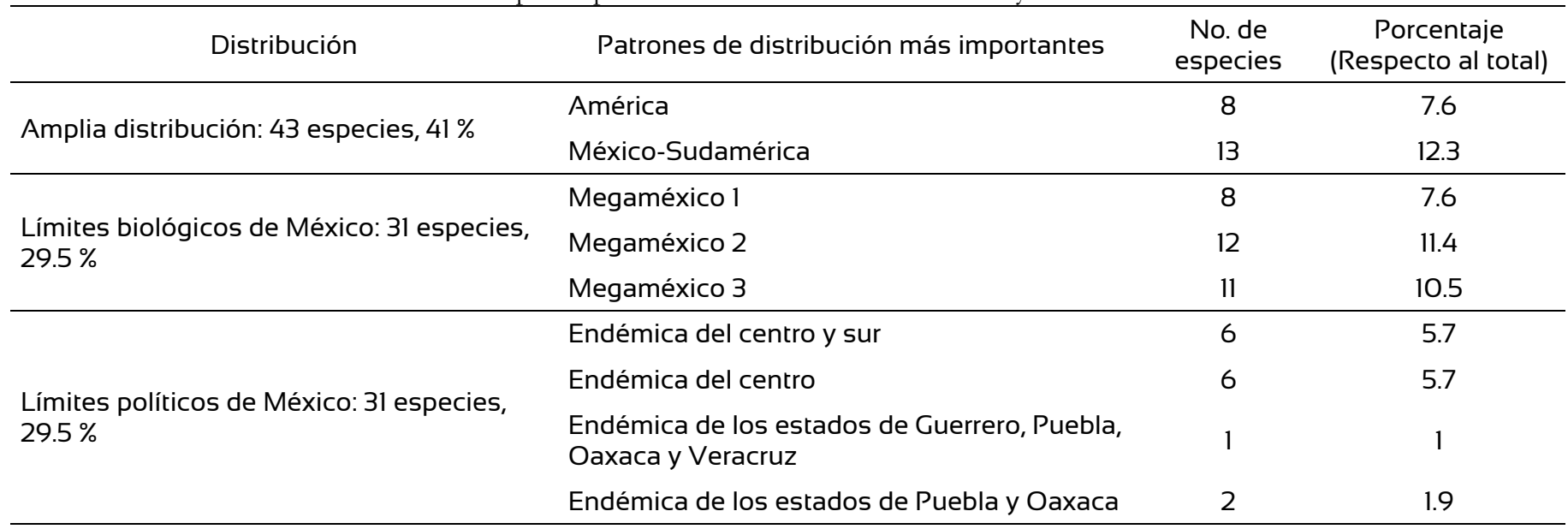


TABLA 4. Índices de diversidad

\begin{tabular}{lcc} 
& Barranca Malinalli & Barranca El Conde \\
\hline Índice de Margalef & 4.98 & 3.97 \\
Índice de Pielou & 0.85 & 0.94 \\
Índice de Simpson & 0.07 & 0.15 \\
Índice de Sorensen & & 0.49 \\
\hline
\end{tabular}

La diversidad de epífitas en la zona de estudio concuerda con algunos hallazgos para bosques templados (CabreraLuna, Huerta-Cantera, Salinas-Soto y Olvera-Valerio, 2015), aunque disminuye respecto a otros registros en bosques de Quercus (Ceja-Romero et al., 2010). El bajo registro podría deberse a que la barranca Malinalli, aunque ubicada a 2060 m s.n.m., se encuentra en una zona seca colindante con matorral xerófilo. La barranca El Conde, aunque más húmeda por su cercanía a la Malinche y por su mayor altitud, tiende a tener menos epifitismo por encontrarse dentro de asentamientos humanos, donde experimenta extracción de especímenes. Sin embargo, esta práctica podría contribuir a la permanencia de este relicto de bosque mediante un aprovechamiento sostenible.

De acuerdo con los patrones de distribución geográfica de la flora de las dos barrancas, $70 \%$ de las familias son de amplia distribución, 25\% de zonas tropicales y subtropicales y $5 \%$ de afinidad boreal. De acuerdo con Rzedowski (1991a), esto se explica en tanto que muchos elementos de la flora mexicana, o sus antecesores, arribaron al territorio desde otras partes del continente o del mundo. En cuanto a géneros, $57 \%$ son de amplia distribución y 43\% son americanos con 15\% de distribución de México a Centroamérica. Este porcentaje de géneros americanos menor a 50\%, en lo general, está en línea con las estimaciones de que son los que en su mayoría componen la flora en México (Villaseñor, 2004). Dicho porcentaje se debe muy posiblemente a que las perturbaciones humanas, sobre todo en la barranca El Conde, han facilitado el arribo y establecimiento de especies pertenecientes a géneros de amplia distribución.
Los patrones de distribución de las especies de la zona de estudio dan cuenta de una flora con el dominio de elementos americanos (86\%), de los cuales un alto porcentaje corresponde a endemismos para México hasta en 59\%. Esto concuerda con la aseveración de Rzedowski (1991b) de que, en los bosques de encino mexicanos, el predominio de especies americanas y endémicas es de aproximadamente $70 \%$ y de que alrededor de $50 \%$ de la flora en México es endémica para el territorio nacional, siendo el bosque de Quercus uno de los centros importantes de endemismo y diversificación vegetal. De acuerdo con De Luna (1985) y Rzedowski (1991a), la riqueza de endemismos en las zonas montañosas de México se debe al aislamiento ecológico que derivó en una larga e intensa evolución in situ, a una gran heterogeneidad ambiental y a una compleja historia geológica del país, todo lo cual contribuyó a la especiación e hibridación de especies (Morrone, 2010).

El análisis de afinidades muestra que la flora de las barrancas presenta una importante relación florística con Centroamérica (24\%). Sin embargo, existe también un importante componente de elementos de afinidad boreal $(18 \%)$. Esta presencia de elementos holárticos y neotropicales se explica por migraciones, en el pasado geológico, de plantas de diversas procedencias, al ser México un puente continental entre América del Norte y América del Sur (Rzedowski, 1988). Sin embargo, la mayor afinidad con Centroamérica es acorde con la aseveración de que, en la flora de la República Mexicana, los elementos de afinidad meridional son proporcionalmente los más importantes (Rzedowski, 1988). 
La composición y riqueza del bosque de Quercus de las barrancas estudiadas demuestra, en concordancia con lo encontrado por Vibrans (1997), “...que aún ambientes muy alterados en México pueden poseer una diversidad biológica considerable", lo cual se hace evidente en las ocho especies de encinos registradas para ambas barrancas y que se presentan en su totalidad en la barranca El Conde. Esto, a pesar de que esta última está sometida a fuertes presiones antropogénicas que han significado la fragmentación del bosque de Quercus - con el desmonte de algunas zonas que actualmente presentan vegetación secundaria en variados estados de sucesión - la contaminación, la introducción de especies exóticas y el quedar constreñida a un mínimo pues no existe zona de amortiguamiento entre la barranca y la zona habitacional que la circunda.

De acuerdo con los resultados arrojados por los índices de Margalef, de Pielou y de Simpson, ambas barrancas presentaron una diversidad alfa de mediana a alta, lo que confirma que se está ante un relicto florístico con un alto valor de biodiversidad. Esto en comparación con la baja diversidad específica encontrada por Mora-Don Juan, Burbano-Vargas, Méndez-Osorio y Castro-Rojas (2017), para bosques de encino de la Sierra Madre del Sur, de acuerdo con el índice de Margalef (0.18); con la diversidad alta señalada por García, Narváez, Olivas y Hernández (2019), para bosques de pino-encino de Chihuahua, conforme al índice de Pielou (0.75 y 0.77); y con la baja diversidad observada por Saavedra-Romero, Hernández-de la Rosa, Alvarado-Rosales, Martínez-Trinidad y VillaCastillo (2019), para el bosque urbano de San Juan de Aragón de la CDMX, de acuerdo con el índice de Simpson (0.85).

Conforme a los índices de Margalef y de Simpson encontrados en este estudio, la barranca Malinalli presentó mayor diversidad, lo cual concuerda con el hecho de que es la que menores impactos antropogénicos ha sufrido, a que presenta menor número de especies introducidas (solo se registraron dos en contraste con las 14 registradas para la barranca El Conde) y a que colinda con zonas de matorral xerófilo, lo que hace que se presenten elementos propios de aquel tipo de vegetación intercalados en los claros dentro el bosque de Quercus.

En cuanto a la diversidad beta, el resultado arrojado por el índice de Sorensen sugirió una similitud florística entre ambas barrancas de 44\%. La heterogeneidad de 56\% entre Malinalli y El Conde podría deberse a la distancia geográfica entre ellas, a los impactos antrópicos negativos sobre la barranca El Conde, a los distintos tipos de suelo y a las diferencias en temperatura y precipitación medias anuales. Este dato expresa una diversidad de la zona por encima de 50\%, lo que se acerca al hecho de que México es considerado un país con una gran diversidad beta o "con una alta tasa de recambio de especies a lo largo de un gradiente de ambientes" (Durand y Neyra, 2010).

\section{CONCLUSIONES}

El presente estudio permitió acercarse a la composición del relicto de bosque de encino de dos barrancas de la ciudad de Puebla, dando cuenta de una importante riqueza de especies (105), géneros (81) y familias (40), un alto componente endémico y dos especies en la categoría de amenazadas, de acuerdo con la NOM-059-SEMARNAT2010. Asimismo, la biodiversidad y heterogeneidad de estos relictos, a pesar de los impactos antrópicos que experimenta la barranca El Conde, reflejan su valor en el contexto de una ciudad carente de espacios arbolados. Lo anterior constituye un motivo para proponer algunas medidas de conservación: (1) asegurar la permanencia de los encinares de las barrancas, previniendo las acciones de tala y extracción de especímenes, a través de monitoreos y difusión de sus beneficios hacia el colectivo social; (2) profundizar estudios sobre su composición en cuanto a las plantas vasculares y extenderlo hacia otro tipo de organismos como las briophytas, hongos y líquenes; así como analizar las características estructurales del bosque y la ecología de la comunidad, a fin de contar con un diagnóstico como paso previo para un programa de restauración; (3) ampliar los estudios hacia todo el sistema de barrancas que entrecruza la ciudad, como un paso que posibilite su reconocimiento como parte del equipamiento de áreas verdes de la ciudad y su creación como área de 
conservación. Esto último es fundamental debido a que no hay dentro de la urbe espacio disponible para zonas de preservación ecológica excepto las barrancas, a que la biodiversidad de las barrancas es contrastante con la pobre diversidad específica de las áreas verdes de la misma (Gutiérrez, 2013) y a que los encinares de las barrancas podrían constituirse en una poza génica y banco de germoplasma nativo in situ y ex situ, como base para el aprovisionamiento de las áreas arboladas de la ciudad y para fomentar las asociaciones ecológicas originarias. Todo esto como una estrategia para conservar los servicios ecosistémicos que las barrancas proporcionan a la ciudad y para promover la identidad de la población con su entorno original.

\section{REFERENCIAS}

Alcaraz A., F. J. (1999). Manual de teoría y práctica de Geobotánica. Murcia, España: ICE Universidad de Murcia y Diego Marín.

Angiosperm Philogeny Group [APG]. (2016). An update of the Angiosperm Philogeny Group classification for the orders and families of flowering plants: APG IV. Botanical Journal of the Linnean Society, 181(1), 1-20. doi: 10.1111/boj.12385.

Ayuntamiento de la ciudad de Puebla (2016). Inventario municipal de áreas verdes (IMAV). Recuperado de http://gobiernoabierto.pueblacapital.gob.mx/transparencia_file /sdus/2015/77.fracc41a/sdus.77.41a.imav.2015.pdf

Cabrera-Luna, J. A., Huerta-Cantera, H. E., Salinas-Soto, P., \& OlveraValerio, D. (2015). Flora y vegetación de la sierra El Rincón, Querétaro y Michoacán, México. Botanical Sciences, 93(3), 615-632. doi: $10.17129 /$ botsci.168

Calderón de Rzedowski, G., \& Rzedowski, J. (2001). Flora fanerogámica del Valle de México. México: Instituto de Ecología A.C., Comisión Nacional para el Conocimiento y Uso de la Biodiversidad.

Ceja-Romero, J., Mendoza-Ruiz, A., López-Ferrari, A. R., Espejo-Serna, A., Pérez-García, B., \& García-Cruz, J. (2010). Las epífitas vasculares del estado de Hidalgo, México: diversidad y distribución. Acta Botanica Mexicana, 93, 1-39. doi: 10.21829/abm93.2010.274

Cronquist, A. (1981). An integrated system of classification of flowering plants. New York, USA: Columbia University Press.

De Luna, E. (1985). Afinidades fitogeográficas de los musgos de los extremos del Eje Neovolcánico, México. Biótica, 10, 235-255.
Durand, L., \& Neyra, L. (2010). La diversidad biológica de México: ecosistemas, especies y genes. En: V. M. Toledo (Ed.), La biodiversidad de México (pp. 12-36). CDMX, México: Fondo de Cultura Económica.

Font, Q. P. (2000). Diccionario de Botánica. Barcelona, España: Ediciones Península.

García G., S. A., Narváez F., R., Olivas G., J. M., \& Hernández S., J. (2019). Diversidad y estructura vertical del bosque de pino-encino en Guadalupe y Calvo, Chihuahua. Revista Mexicana de Ciencias Forestales, 10(53), 41-63. doi: 10.29298/rmcf.v10i53.173

Gutiérrez P., V. (2013). Los espacios públicos abiertos de la zona de monumentos del centro histórico de la ciudad de Puebla. Tesis de maestría, Benemérita Universidad Autónoma de Puebla, Puebla, México.

International City/County Management Association [ICMA]. (2017). Reporte de resultados SINDES (Sistema de Indicadores de Desempeño), $2^{\circ}$ semestre 2017. Recuperado de http://www.icmaml.org/wpcontent/uploads/2018/05/Reporte\%20resultados\%20SINDES $\% 202$ do\%20semestre\%202017\%20vf.pdf

Instituto de Geografía-UNAM. (1989). Diccionario geomorfológico. CDMX, México: Publicaciones UNAM.

Instituto Nacional de Estadística y Geografía [Inegi]. (2010). Compendio de información geográfica municipal de los Estados Unidos Mexicanos Puebla Puebla. México: Inegi.

López-Domínguez, J. C., \& Acosta P., R. (2005). Descripción del Parque Nacional Malinche. En J. A. Fernández F., \& J. C. LópezDomínguez (Eds.), Biodiversidad del Parque Nacional MalincheTlaxcala, México (pp. 3-24). Tlaxcala, México: Coordinación General de Ecología del Gobierno del Estado de Tlaxcala.

Lot, A., \& Chiang, C. (1986). Manual de herbario, administración y manejo de colecciones, técnicas de recolección y preparación de ejemplares botánicos. CDMX, México: Consejo Nacional de la Flora de México, A.C.

McVaugh, R. (1992). Gymnosperms and pteridophytes. Flora NovoGaliciana, 17, 1-467.

Mickel, J. T., \& Smith, A. R. (2004). The pteridophytes of México. Memoirs of the New York Botanical Garden, 88, 1-1092.

Miranda, F. (1947). Estudio sobre la vegetación de México. V. Rasgos de la vegetación de la Cuenca del Río Balsas. Revista de la Sociedad Mexicana de Historia Natural, 8, 95-114.

Missouri Botanical Garden (2014). Tropicos.org. Recuperado de http://www.tropicos.org

Mora-Don Juan, C. A., Burbano-Vargas, O. N., Méndez-Osorio, C., \& Castro-Rojas, D. F. (2017). Evaluación de la biodiversidad y caracterización estructural de un Bosque de Encino (Quercus L.) 
en la Sierra Madre del Sur, México. Revista Forestal Mesoamericana Kurú, 14(35), 68-75. doi: 10.18845/rfmk.v14i35.3154

Moreno, C. E. (2001). Métodos para medir la biodiversidad, vol.1. Zaragoza, España: M y T-Manuales y Tesis SEA.

Morrone, J. J. (2010). Fundamental biogeographic patterns across the Mexican Transition Zone: an evolutionary approach. Ecography, 33, 355-361. doi: 10.1111/j.1600-0587.2010.06266.x.

National Museum of Natural History [NMNH]. (2019). Encyclopedia of Life. Recuperado de https://www.eol.org

Nixon, K. C. (1993). The genus Quercus in Mexico. En T. P. Ramamoorthy, R. Bye, A. Lot, \& J. Fa (Eds.), Biological diversity of Mexico: origins and distribution (pp. 447-458). Nueva York: Oxford University Press.

Nixon, K. C. (2009). An overview of Quercus. Classification and phylogenetics with comments on differences in wood anatomy. Recuperado de https://www.texasoakwilt.org/NOWS/conference_assets/conferencepapers/Nixon.pdf

Nowak, D., Crane, D., \& Stevens, J. (2006). Air pollution removal by urban trees and shrubs in the United States. Urban Forestry and Urban Greening, 4(3-4), 115-123. doi: 10.1016/j.ufug.2006.01.007

Rodríguez A., M., Coombes, A. J., \& Jiménez R., J. (2009). Plantas silvestres de Puebla. Puebla, México: Benemérita Universidad Autónoma de Puebla.

Rzedowski, J. (1988). Vegetación de México. CDMX, México: Escuela Nacional de Ciencias Biológicas-IPN-Limusa.

Rzedowski, J. (1991a). Diversidad y orígenes de la flora fanerogámica de México. Acta Botanica Mexicana, 14, 3-21. doi: 10.21829/abm14.1991.611

Rzedowski, J. (1991b). El endemismo en la flora fanerogámica mexicana: una apreciación analítica preliminar. Acta Botanica Mexicana, 15, 47-64. doi: 10.21829/abm15.1991.620

Saavedra-Romero, L. de L., Hernández-de la Rosa, P., Alvarado-Rosales, D., Martínez-Trinidad T., \& Villa-Castillo J. (2019). Diversidad, estructura arbórea e índice de valor de importancia en un bosque urbano de la Ciudad de México. Polibotánica, 47, 25-37. doi: $10.18387 /$ polibotanica.47.3

Sánchez-Rodríguez, E. V., López-Mata, L., García-Moya, E. y CuevasGuzmán, R. (2003). Estructura, composición florística y diversidad de especies leñosas de un bosque mesófilo de montaña en la Sierra de Manantlán, Jalisco. Boletín de la Sociedad Botánica de México, 73, 17-34.

Secretaría de Medio Ambiente y Recursos Naturales [Semarnat], (2010). Norma Oficial Mexicana NOM-059-SEMARNAT-2010, Protección ambiental-Especies nativas de México de flora y fauna
silvestres-Categorías de riesgo y especificaciones para su inclusión, exclusión o cambio-Lista de especies en riesgo. Recuperado de: http://dof.gob.mx/nota_detalle_popup.php?codigo $=5173091$

Secretaría de Medio Ambiente y Recursos Naturales [Semarnat] y Comisión Nacional Forestal [Conafor], (2013). Inventario Estatal Forestal y de Suelos-Puebla 2013. México: Semarnat y Conafor.

Sosa, A. (1927). Los bosques de la Malinche. En J. A. Fernández F., \& J. C. López-Domínguez (2005). Biodiversidad del Parque Nacional Malinche-Tlaxcala, México. Tlaxcala, México: Coordinación General de Ecología del Gobierno del Estado de Tlaxcala.

Spurr, S. H. (1964). Forest ecology (1a ed.). Nueva York, EUA: Ronald Press Company.

The Plant List. (2013). Versión 1.1. Recuperado de http://www.theplantlist.org

Valencia A., S. (2004). Diversidad del género Quercus (Fagaceae) en México. Boletin de la Sociedad Botánica Mexicana, 75, 33-53. doi: $10.17129 /$ botsci.1692

Valencia, A. S. (2010). Notes of the genus Quercus in Mexico. International Oaks, 21, 100-120.

Vibrans, H. (1997). Lista florística comentada de plantas vasculares silvestres en San Juan Quetzalcoapan, Tlaxcala, México. Acta Botanica Mexicana, 38, 21-67. doi: 10.21829/abm38.1997.773

Vibrans, H. (2006). Malezas de México. Recuperado de http://www.malezasdemexico.net

Villaseñor, J. L. (2003). Diversidad y distribución de las Magoliophyta de México. Interciencia, 28(3), 160-167.

Villaseñor, J. L. (2004). Los géneros de plantas vasculares de la flora de México. Boletín de la Sociedad Botánica Mexicana, 75, 105-135. doi: 10.17129/botsci.1694

Villaseñor, J. L., Ortiz, E., \& Redonda-Martínez, R. (2008). Catálogo de autores de plantas vasculares de México. CDMX, México: Universidad Nacional Autónoma de México/ Comisión Nacional para el Conocimiento y Uso de la Biodiversidad.

Villaseñor, J. L., \& Ortiz, E. (2007). La familia Asteraceae en la Faja Volcánica Transmexicana. En I. Luna V., J. J. Morrone, \& D. Espinoza O. (Eds.). Biodiversidad de la Faja Volcánica Transmexicana (pp. 289-310). CDMX, México: Universidad Nacional Autónoma de México.

Villaseñor, J. L., \& Ortiz, E. (2014). Biodiversidad de las plantas con flores (División Magnoliophyta) en México. Revista Mexicana de Biodiversidad, 85, 134-142. doi: 10.7550/rmb.31987

Zepeda Gómez, C., Burrola Aguilar, C., White Olascoaga, L., \& Rodríguez Soto, C. (2017). Especies leñosas útiles de la selva baja 
Madera y Bosques vol. 27, núm. 1, e2712113 Primavera 2021

caducifolia en la Sierra de Nanchititla, México. Madera y Bosques, 23(3), 101-119. doi: 10.21829/myb.2017.2331426

Manuscrito recibido el 23 de enero 2020

Aceptado el 18 de junio de 2020

Publicado el 9 de julio de 2021
Este documento se debe citar como:

Gutiérrez Pacheco, V, Silva Gómez, S. E., \& y Varela Olguín, L. L. (2021). Flora del bosque de encino (Quercus: Fagaceae) de dos barrancas de la ciudad de Puebla, México. Madera y Bosques, 27(1), e2712113. doi: 10.21829/myb.2021.2712113

Madera y Bosques por Instituto de Ecología, A.C.

se distribuye bajo una Licencia Creative Commons Atribución-NoComercialCompartirlgual 4.0 Internacional. 